\title{
Clinical and imaging features of 112 patients with irregular and flat bone osteosarcoma
}

\author{
Zhendong Luo ${ }^{1 \#}$, Jing $\mathrm{Li}^{2 \#}$, Genggeng Qin ${ }^{3}$, Hui Zeng ${ }^{3}$, Zilong $\mathrm{He}^{3}$, Derun Pan ${ }^{3}$, Yulin $\mathrm{Li}^{4}$, Weiguo Chen ${ }^{3}$, \\ Xinping Shen ${ }^{1}$
}

${ }^{1}$ Department of Radiology, The University of Hong Kong-Shenzhen Hospital, Shenzhen, China; ${ }^{2}$ Department of Radiology, State Key Laboratory of Oncology in South China, Collaborative Innovation Center for Cancer Medicine, Sun Yat-sen University Cancer Center, Guangzhou, China; ${ }^{3}$ Department of Radiology, Nanfang Hospital, Southern Medical University, Guangzhou, China; ${ }^{4}$ Department of Radiology, Peking University Shenzhen Hospital, Shenzhen, China

Contributions: (I) Conception and design: Z Luo, J Li, X Shen, W Chen; (II) Administrative support: None; (III) Provision of study materials or patients: None; (IV) Collection and assembly of data: Z He, H Zeng, D Pan, Y Li; (V) Data analysis and interpretation: G Qin, Z Luo, J Li; (VI) Manuscript writing: All authors; (VII) Final approval of manuscript: All authors.

\#These authors contributed equally to this work.

Correspondence to: Weiguo Chen. Department of Radiology, Nanfang Hospital, Southern Medical University, No. 1838, Guangzhou Avenue North, Baiyun District, Guangzhou 510000, China. Email: chen1999@smu.edu.cn; Xinping Shen. Department of Radiology, The University of Hong Kong-Shenzhen Hospital, No. 1, Haiyuan Road, Futian District, Shenzhen 518000, China. Email: szshenxinping@163.com.

Background: This study evaluated the clinical characteristics and imaging findings of 112 patients with irregular and flat bone osteosarcoma (IFBO).

Methods: The age, gender, location, tumor size, density and signal intensity, osteoid matrix, periosteal reaction, and histological subtypes were analyzed for 112 patients with IFBO.

Results: A total of 112 patients with IFBO, including 64 males and 48 females, with a mean age of 34.8 years were enrolled in this study. Over half of the tumors $(54.5 \%)$ were detected in the craniofacial region and the skull (24 in the maxilla bone, 17 in the mandible bone, 11 in the sphenoid bone, 7 in the temporal bone, 1 in the frontal bone, and 1 in the occipital bone). Other tumor locations included the pelvic region $(20.5 \%$; 20 in the ilium and 3 in the pubis), the chest $(18.8 \% ; 11$ in the scapula, 7 in the ribs, and 3 in the clavicle), and the vertebrae $(6.3 \% ; 3$ in the thoracic spine, 2 in the lumbar spine, 1 in the sacrum, and 1 in the cervical spine). Transarticular extension occurred in 11 of the 23 pelvic cases (47.8\%), primarily involving the sacroiliac joint (90.9\%; 10 of 11$)$. Six cases $(6 / 7 ; 85.7 \%)$ of vertebral osteosarcoma arose from the transverse process and the pedicle, and $1(1 / 7 ; 14.3 \%)$ arose from the sacral tuberosity and the ala, with partial vertebral body involvement. Additionally, 27 patients (24.1\%) presented with secondary osteosarcoma related to prior radiotherapy, and $2(1.8 \%)$ were associated with osteoblastoma and fibrous dysplasia. Histological examination revealed high-grade tumors in $88(78.6 \%)$ cases. The tumors presented as softtissue masses with a diameter of $7.5 \pm 3.2 \mathrm{~cm}$. A total of 91 patients underwent X-ray examination and/or computed tomography (CT) examinations. The osteoid matrix was detected in 84 patients $(84 / 91 ; 92.3 \%)$. A periosteal reaction was detected in 56 cases $(56 / 91 ; 61.5 \%)$, including a lamellar periosteal reaction in 10 patients (11.0\%) and a spiculated periosteal reaction in 46 cases (50.5\%). All 74 cases who underwent magnetic resonance imaging (MRI) examinations presented with heterogeneous masses in the surrounding soft tissue. Enhancement was homogenous in 12 cases (18.5\%) and heterogeneous in 53 cases $(81.5 \%)$. Peripheral rim enhancement was observed in 10 cases (13.5\%).

Conclusions: IFBO should be considered when diagnosing patients over 30 years of age who exhibit osteoid matrix in bone lesions. Maxillofacial osteosarcoma is commonly associated with a history of radiation 
exposure. Pelvic osteosarcoma is more likely to invade the sacroiliac joint. Vertebral osteosarcoma frequently arises in the transverse process and pedicle, with partial body involvement.

Keywords: Osteosarcoma; irregular bone; flat bone; computed tomography (CT); magnetic resonance imaging (MRI)

Submitted Apr 12, 2021. Accepted for publication Nov 24, 2021.

doi: 10.21037/qims-21-393

View this article at: https://dx.doi.org/10.21037/qims-21-393

\section{Introduction}

Osteosarcoma is the most common childhood bone cancer. Approximately 1,000 new cases of osteosarcoma are diagnosed each year in the United States (1). Osteosarcomas frequently affect long bones but can also occur in various flat bones and soft tissues (2). Flat bone osteosarcoma (FBO) accounts for approximately $4.7 \%$ of all osteosarcomas and the incidence increases with age (2-5). To date, a limited number of studies have focused on the clinical and histopathological prognostic factors influencing irregular and flat bone osteosarcomas (IFBOs) (4,6-8). Although there have been previous case reports and case studies focusing on IFBO imaging in specific body regions, there is a paucity of comprehensive studies detailing the clinical and imaging features of IFBO (9-12). In contrast to osteosarcoma of the extremities, the low incidence of IFBO is associated with significant diagnostic challenges. This current study analyzed the imaging features of IFBOs.

\section{Methods}

This study was conducted in accordance with the Declaration of Helsinki (as revised in 2013) and was approved by the ethics board of the hospital. Individual patient consent for this retrospective analysis was waived. A total of 398 patients with osteosarcoma underwent preoperative imaging, consisting of computed tomography (CT) and/or magnetic resonance imaging (MRI), and were treated in the orthopedic departments of four institutions (the University of Hong Kong-Shenzhen Hospital, Sun Yat-sen University Cancer Center, Nanfang Hospital and Peking University Shenzhen Hospital) from June 2005 to December 2020. All available electronic medical databases were searched, and 112 patients diagnosed with IFBO either by surgical resection $(n=42)$ or by CT- or ultrasoundguided needle biopsy ( $\mathrm{n}=70)$ were identified. The diagnoses included primary or secondary bone osteosarcomas arising
Table 1 Technical parameters of the computed tomography scanner

\begin{tabular}{lc}
\hline Parameters & Value \\
\hline Voltage $(\mathrm{kV})$ & 120 \\
Exposure $(\mathrm{mAs})$ & $200-300$ \\
Thickness $(\mathrm{mm})$ & $1-3$ \\
Pitch & 1.5 \\
Image reconstruction matrix & 2 \\
Reconstruction kernel & Standard \\
Reconstructed slice thickness $(\mathrm{mm})$ & $3-5$ \\
\hline
\end{tabular}

from the irregular and flat bones of the craniofacial region and skull (mandible, maxilla, sphenoid, frontal, temporal, and occipital bones), the pelvic region (ilium and pubis), the chest (rib, clavicle, and scapula), and the vertebrae (cervical, thoracic, lumbar, and sacrum). The diagnoses of IFBO were made based on clinical and imaging findings and were confirmed by histopathological examinations. Pathological findings were reviewed by a pathologist with 10 years of experience in bone pathology.

The inclusion criteria were as follows: patients with a diagnosis of IFBO confirmed by histology; no history of prior treatments; and patients who underwent imaging examinations. The tumor size was determined via a threedimensional (3D) measurement of the tumor with CT or MRI.

The imaging examinations were performed using a 16or 64-slice CT system (Siemens SOMATOM Definition AS 64, Siemens, Berlin, Germany; Toshiba Aquilion TM64, Toshiba, Tokyo, Japan; or GE Lightspeed Ultra 16, GE Health care, Chicago, IL, USA) or a 1.5 or $3.0 \mathrm{~T}$ MRI scanner (Siemens Magnetom Avanto 1.5, Siemens; Siemens Magnetom Trio Tim 3.0, Siemens; or GE Signa Excite HD 3.0, GE Health care).

The main CT and MRI parameters of each protocol are described in Tables 1,2. Patient demographics, 
Table 2 Technical parameters of the magnetic resonance imaging scanner

\begin{tabular}{lccccccc}
\hline Sequence & Category & TR $(\mathrm{ms})$ & TE $(\mathrm{ms})$ & FOV $(\mathrm{mm} \times \mathrm{mm})$ & Matrix & Intersection gap $(\mathrm{mm})$ & Slice thickness $(\mathrm{mm})$ \\
\hline T1WI & FSE & $400-650$ & $10-25$ & $180 \times 180-380 \times 380$ & $288 \times 256-416 \times 384$ & 0 & $3-7$ \\
T2WI & FSE & $2,210-6,367$ & $65-140$ & $180 \times 180-380 \times 380$ & $288 \times 256-416 \times 384$ & 0 & $3-7$ \\
T2WI-FS & FSE & $2,210-6,367$ & $65-140$ & $180 \times 180-380 \times 380$ & $288 \times 256-416 \times 384$ & 0 & $3-7$ \\
CE-T1WI & FSE & $400-650$ & $10-25$ & $180 \times 180-380 \times 380$ & $288 \times 256-416 \times 384$ & 0 & $3-7$ \\
CE-T1WI-FS & FSE & $457-798$ & $12-25$ & $180 \times 180-380 \times 380$ & $288 \times 256-416 \times 384$ & 0 & $3-7$ \\
\hline
\end{tabular}

TR, repetition time; TE, echo time; FOV, field of view; T1WI, T1-weighted imaging; T2WI, T2-weighted imaging; FS, fat saturation; FSE, fast spin echo; CE, contrast-enhanced.

imaging features, and clinical data were recorded. All imaging data were acquired using the picture archiving and communication system (PACS) by two experienced radiologists with over 10 years of experience in radiological research on bone tumors. All images were assessed with an emphasis on the following criteria: (I) tumor location; (II) tumor density; (III) osteoid matrix; (IV) periosteal reaction; (V) tumor size (determined via a 3D measurement of the tumor with CT or MRI); (VI) tumor margin; and (VII) pattern of enhancement of the lesion (homogeneous or heterogeneous). A nonenhanced portion in the center of the tumor was regarded as a necrotic or cystic component. An enhancing peripheral rim of variable thickness in the tumor was categorized as a peripheral enhancement. Importantly, items (II), (III), and (IV) were recorded on radiographs and/ or CT scans, and items (V), (VI), and (VII) were recorded on CT and/or MRI scans. All cases were histologically reviewed, and the diagnoses were made according to the 2013 World Health Organization (WHO) classification of soft tissue and bone tumors (13), which lists multiple histological subtypes of IFBO, including conventional (osteoblastic, fibroblastic, and chondroblastic), small-cell, giant cell-rich, well-differentiated variants.

\section{Results}

The patients' clinical features are summarized in Table 3. There were 112 patients in the study cohort, including 64 (57.1\%) males and 48 (42.9\%) females, with a mean age of 34.8 years (range, 9-73 years). Over half of the tumors $(54.5 \%)$ were detected in the craniofacial region and skull (including 24 in the maxilla bone, 17 in the mandible bone, 11 in the sphenoid bone, 7 in the temporal bone, 1 in the frontal bone, and 1 in the occipital bone). Other tumor locations included the pelvic region (20.5\%; including 20 in the ilium and 3 in the pubis), the chest (18.8\%; including 11 in the scapula, 7 in the ribs, and 3 in the clavicle), and the vertebrae $(6.3 \%$; including 3 in the thoracic spine, 2 in the lumbar spine, 1 in the sacrum, and 1 in the cervical spine). Out of the 23 patients with pelvic osteosarcoma, transarticular extension occurred in 11 cases $(47.8 \%), 10$ of which involved the sacroiliac joint $(90.9 \%)$. Six cases of vertebral osteosarcomas arose from the transverse process and the pedicle, and 1 arose from the sacral tuberosity and the ala, with partial vertebral body involvement.

The histological features of the patients are presented in Table 4. Histological examination revealed that 7 (6.3\%) patients presented with low-grade tumors, 88 (78.5\%) had high-grade tumors, and 17 (15.2\%) had tumors with an unknown grade. Histological subtypes were reported for high-grade cases, including 46 (41.1\%) patients with osteoblastic type, 24 (21.4\%) with chondroblastic type, 11 $(9.8 \%)$ with fibroblastic type, and 4 (3.6\%) with small-cell osteosarcoma. In addition, 3 (2.7\%) patients had giant cellrich osteosarcoma.

Imaging examinations were performed, included 37 cases of radiography $(37 / 112 ; 33 \%), 83$ CT scans $(83 / 112$; $74.1 \%)$, and 74 MRI scans $(74 / 112 ; 66.1 \%)$. Of the CT scans, $70(70 / 83 ; 84.3 \%)$ were contrast-enhanced CT examinations and $13(13 / 83 ; 15.7 \%)$ were non-contrastenhanced CT examinations. Contrast-enhanced MRI scans were performed on 65 of the $74(87.8 \%)$ patients.

The imaging features of patients with IFBO are summarized in Table 5. Ninety-one patients underwent radiographs or CT examinations. Among them, 23 cases had osteolytic bone lesions, 32 had osteoblastic bone lesions, and 36 presented with mixed osteolytic/osteoblastic bone lesions. The osteoid matrix was detected in 84 of the 91 cases $(92.3 \%)$ (Figures 1-5). A periosteal reaction was found in 56 of the 91 cases $(61.5 \%)$, including a lamellar 
Table 3 Clinical features of all 112 irregular and flat bone osteosarcoma (IFBO) patients

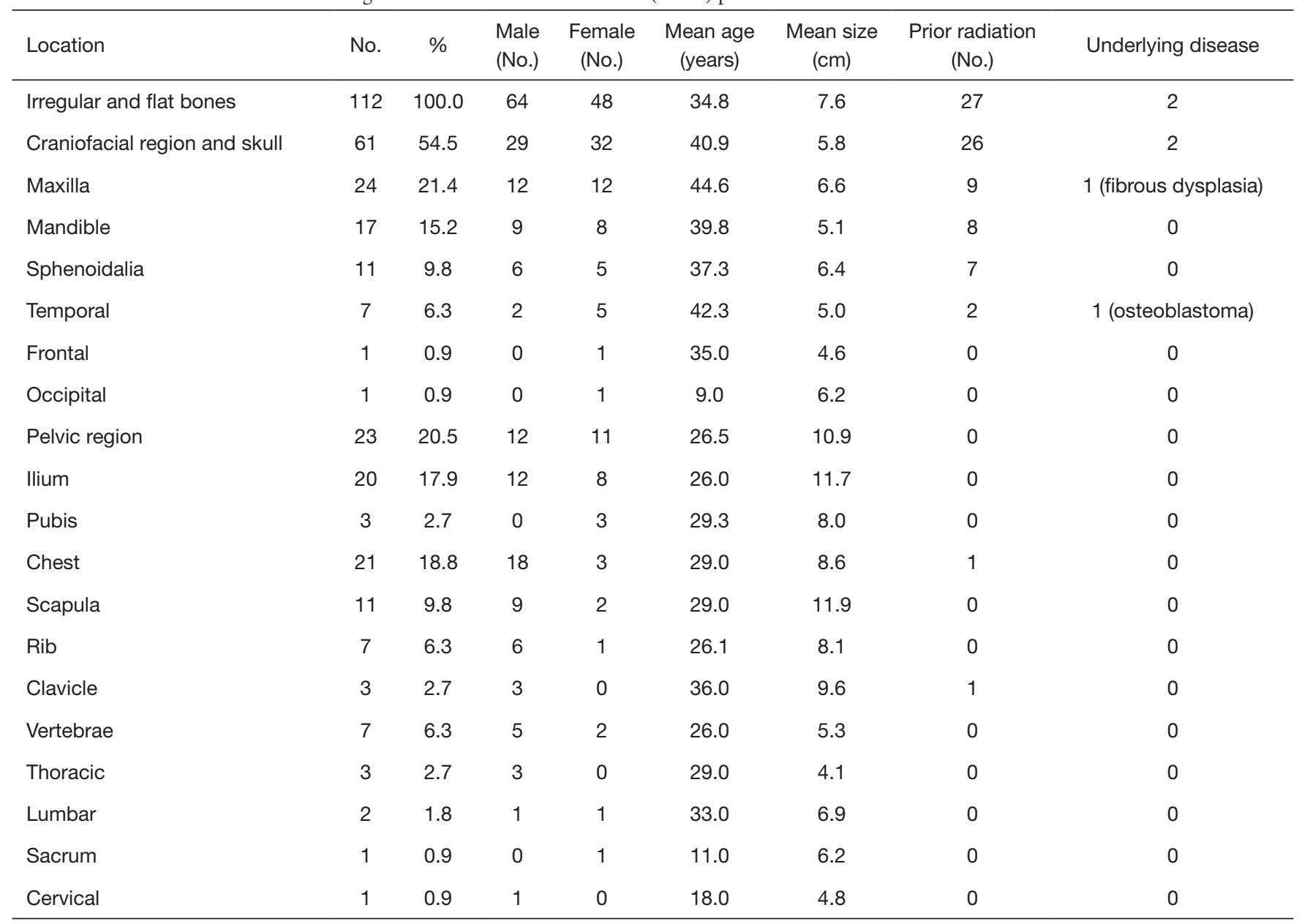

periosteal reaction in $10(11.0 \%)$ cases and a spiculated periosteal reaction in $46(50.5 \%)$ cases.

On radiography, 30 of the $37(81.1 \%)$ cases demonstrated various degrees of bone destruction, and 35 of the 37 (94.6\%) cases showed a high density of the osteoid. In addition, a soft-tissue mass was identified in 26 of the 37 (70.3\%) cases (Figures 3A, 5A,6A).

On CT, all $83(100.0 \%)$ cases showed various degrees of bone destruction, and only 1 case of pathologic fracture was detected. There were 75 (90.4\%) cases with a primary bone tumor extending into the surrounding soft tissues. In addition, 82 of the 83 (98.8\%) cases had heterogeneous soft-tissue attenuation, and only 1 case of homogeneous soft-tissue attenuation was detected. Moreover, 76 of the 83 $(91.6 \%)$ cases had irregular or punctate osteoid matrix in the soft-tissue mass and the area of bone destruction (Figures 1-6).
On MRI, all 74 cases presented with heterogeneous surrounding soft tissue masses. Iso- or hypointensity was recorded on the T1-weighted images, while iso- or hyperintensity was recorded on the $\mathrm{T} 2$-weighted images, and hyperintensity was recorded on the short tau inversion recovery (STIR) sequences (Figures 1-6). Contrastenhanced T1-weighted imaging was performed in 65 of the $74(87.8 \%)$ cases. The mass, destruction of the bone, and soft-tissue tumors exhibited different degrees of contrastenhancement. The enhancement was homogenous in 12 of the $65(18.5 \%)$ cases and heterogeneous in 53 of the 65 (81.5\%) cases. Peripheral rim enhancement was observed in 10 cases $(13.5 \%)$.

\section{Discussion}

In the present study, the incidence of IFBO was higher 
Table 4 Histology of all 112 irregular and flat bone osteosarcomas (IFBOs)

\begin{tabular}{|c|c|c|c|c|c|c|c|c|c|}
\hline Location & \multicolumn{6}{|c|}{ Histological } & \multicolumn{3}{|c|}{ Pathological grade } \\
\hline Irregular and flat bones & 46 & 24 & 11 & 4 & 3 & 17 & 88 & 7 & 17 \\
\hline Craniofacial region and skull & 24 & 12 & 7 & 0 & 2 & 12 & 45 & 4 & 12 \\
\hline Maxilla & 6 & 5 & 5 & 0 & 1 & 5 & 17 & 2 & 5 \\
\hline Sphenoidalia & 5 & 2 & 0 & 0 & 0 & 3 & 7 & 1 & 3 \\
\hline Temporal & 4 & 1 & 0 & 0 & 1 & 0 & 6 & 1 & 0 \\
\hline Frontal & 1 & 0 & 0 & 0 & 0 & 0 & 1 & 0 & 0 \\
\hline Occipital & 1 & 0 & 0 & 0 & 0 & 0 & 1 & 0 & 0 \\
\hline Pubis & 0 & 2 & 1 & 0 & 0 & 0 & 3 & 0 & 0 \\
\hline Chest & 11 & 3 & 2 & 0 & 1 & 2 & 17 & 2 & 2 \\
\hline Scapula & 5 & 1 & 1 & 0 & 0 & 2 & 7 & 2 & 2 \\
\hline Rib & 4 & 1 & 1 & 0 & 1 & 0 & 7 & 0 & 0 \\
\hline Clavicle & 2 & 1 & 0 & 0 & 0 & 0 & 3 & 0 & 0 \\
\hline Vertebrae & 4 & 0 & 1 & 2 & 0 & 0 & 7 & 0 & 0 \\
\hline Thoracic & 3 & 0 & 0 & 0 & 0 & 0 & 3 & 0 & 0 \\
\hline Lumbar & 0 & 0 & 1 & 1 & 0 & 0 & 2 & 0 & 0 \\
\hline
\end{tabular}

(112/398 patients with osteosarcoma) than that reported in previous studies (2-8). This discrepancy may be explained by 2 factors. First, some patients with extremity osteosarcoma, who did not undergo MRI or CT scans before surgery, were excluded from the study. Second, approximately $25 \%$ of the patients in the cohort $(27 / 112$; $24.1 \%$ ) had received prior radiotherapy, and in 26 of these 27 cases $(96.3 \%)$, IFBO was detected in the maxillofacial region. Secondary osteosarcoma is directly related to prior treatments (such as irradiation) or skeletal disorders (such as Paget's disease and fibrous dysplasia) (7), and radiotherapy is an important therapeutic scheme for head and neck cancers, especially nasopharyngeal carcinoma (NPC). The results from our study may be correlated with the high prevalence of NPC in the Asian population, with the nasopharynx and maxillofacial areas being the most irradiated subsites in such an epidemiological scenario. Indeed, 2 patients in our cohort presented with secondary osteoblastoma and fibrous dysplasia, which is consistent with previous findings (14-19).

In our study cohort, the 2 most frequent sites for osteosarcomas were the maxilla in the craniofacial region and the ilium in the pelvic region. Approximately $50 \%$ of all pelvic osteosarcomas with large volumes commonly involved the sacroiliac joint in our study. Previous reports have suggested that rib osteosarcomas are mostly associated with pre-existing lesions, such as Paget's disease or irradiated bone (20). However, this was inconsistent with the findings of the present study, in which none of the 7 patients with osteosarcoma of the ribs had any history of exposure to radiation or Paget's disease. While vertebral osteosarcomas are relatively rare, with a reported incidence of $2-4 \%$ (21), $1.76 \%$ were identified in our study cohort, and the vertebral tumors arose from the transverse process and the pedicle with partial vertebral body involvement, and 
Table 5 Imaging features of 112 irregular and flat bone osteosarcoma (IFBO) patients

\begin{tabular}{|c|c|c|c|c|c|c|c|c|c|c|c|c|}
\hline \multirow{2}{*}{ Location } & \multicolumn{3}{|c|}{ Density (X-ray/CT) } & \multicolumn{2}{|c|}{$\begin{array}{l}\text { Osteoid matrix } \\
\text { (X-ray/CT) }\end{array}$} & \multicolumn{3}{|c|}{$\begin{array}{c}\text { Periosteal reaction } \\
(\mathrm{X}-\text {-ray/CT })\end{array}$} & \multicolumn{2}{|c|}{$\begin{array}{l}\text { Margin definition } \\
\text { (CT/MRI) }\end{array}$} & \multicolumn{2}{|c|}{$\begin{array}{l}\text { Density/SI unenhancec } \\
\text { (necrosis) (CT/MRI) }\end{array}$} \\
\hline & Mixed & Lytic & Osteoid & Yes & No & SPIC & LAM & No & III & Well & Yes & No \\
\hline Craniofacial region and skull & 20 & 11 & 18 & 44 & 5 & 26 & 6 & 17 & 58 & 3 & 48 & 13 \\
\hline Sphenoid & 3 & 2 & 2 & 6 & 1 & 5 & 0 & 2 & 11 & 0 & 6 & 5 \\
\hline Temporal & 2 & 1 & 1 & 4 & 0 & 1 & 1 & 2 & 6 & 1 & 5 & 2 \\
\hline Frontal & 0 & 0 & 1 & 1 & 0 & 0 & 0 & 1 & 1 & 0 & 0 & 1 \\
\hline Pubis & 1 & 2 & 0 & 3 & 0 & 0 & 1 & 2 & 3 & 0 & 2 & 0 \\
\hline Chest & 7 & 4 & 7 & 18 & 0 & 9 & 1 & 8 & 21 & 0 & 21 & 0 \\
\hline Rib & 1 & 2 & 4 & 7 & 0 & 3 & 0 & 4 & 6 & 1 & 7 & 0 \\
\hline Scapula & 5 & 2 & 1 & 8 & 0 & 4 & 0 & 4 & 11 & 0 & 11 & 0 \\
\hline Clavicle & 1 & 0 & 2 & 3 & 0 & 2 & 1 & 0 & 3 & 0 & 3 & 0 \\
\hline Vertebrae & 1 & 3 & 3 & 7 & 0 & 3 & 0 & 4 & 7 & 0 & 6 & 1 \\
\hline Thoracic & 0 & 1 & 2 & 3 & 0 & 2 & 0 & 1 & 3 & 0 & 2 & 1 \\
\hline
\end{tabular}

CT, computed tomography; MRI, magnetic resonance imaging; SPIC, spiculated; LAM, lamellar; SI, signal.

this latter characteristic has not been reported previously.

The age of patients with IFBO reported in the literature ranges from 23 to 28.7 years old $(3,4,8)$. In the present study, the age of patients at onset ranged from 9 to 73 years old. The onset age of most extremity osteosarcomas was children and adolescents (22), and most IFBO (88/112; $78.6 \%$ ) were over 30 years in our study which was higher than that of extremity osteosarcomas. In the current study the patients with mandibular, maxillary, and skull base lesions ranged from 30 to 50 years old, which was consistent with previous reports (11). Studies have mentioned 2 agedependent peaks in patients with osteosarcoma of the pelvic bones, including adolescence (16.8-20 years old) and later adulthood ( $>50$ years old) $(10,23,24)$. However, in the current study, the mean age of patients with osteosarcoma of the pelvic bones was 26.5 years old, and only 2 patients were older than 50 years. The reason for this discrepancy may be related to the different populations that were studied.

In this study cohort, the gender distribution of patients with IFBO indicated a slightly higher proportion of males, with a male to female ratio of $1.33: 1$ compared to a ratio of 1.12:1 reported by Lee et al. (6).

Osteosarcoma typically presents in 3 forms: isolated osteolytic, isolated osteoblastic, or mixed osteolytic/ osteoblastic lesions. The typical radiographic findings of osteosarcomas include 1 or more mixed density shadows, with several banded and patchy areas of osteoid matrix (25). Osteoid matrix was detected in $92.3 \%$ of patients in the current study, which was not significantly different from that 

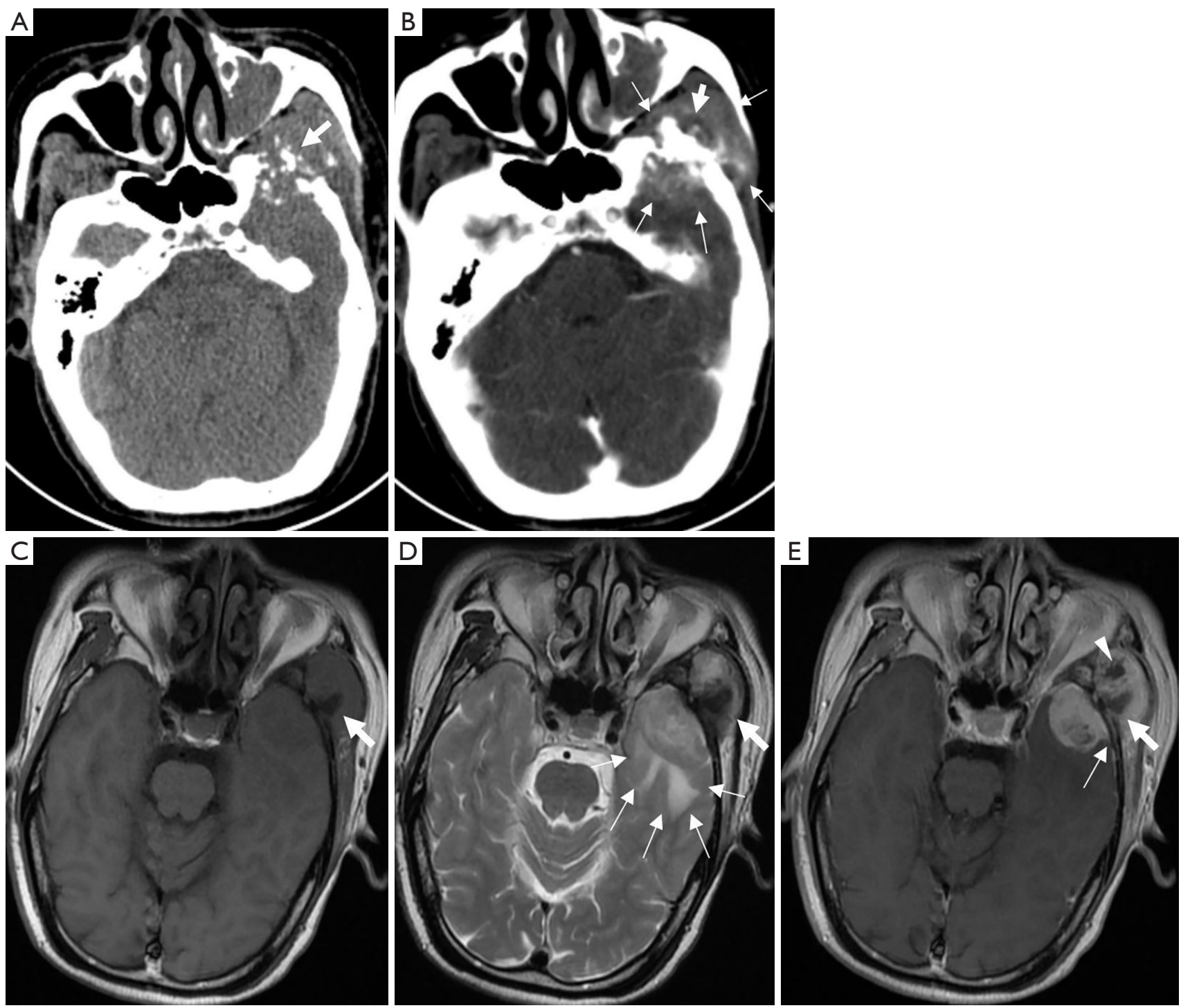

Figure 1 Medical imaging of a 58-year-old male with osteosarcoma of the left temporal bone (histological subtype: osteoblastic). (A) An axial computed tomography (CT) image showing a mixed density mass with cortical destruction at the left temporal bone. Soft tissue mass with a fluffy-like osteoid matrix (thick white arrow) can be observed. The demarcation between the tumor and the left temporal lobe was unclear. (B) A postcontrast axial CT image showing heterogeneous enhancement of the extraosseous soft-tissue extension (thin white arrows) with nonenhanced necrosis (thick white arrow). (C,D) An axial T1-weighted image (C) and a T2-weighted image (D) showing heterogeneous signal intensity with patchy hypointensity (thick white arrows) of the tumor and edema of the left temporal lobe (thin white arrows). (E) An axial T1-weighted contrast-enhanced image showing a heterogeneous, markedly enhanced mass with hypointense osteoid matrix (thick white arrow), non-enhanced necrosis (white arrowhead), and a dural tail sign (thin white arrow).

observed in typical osteosarcomas of the extremities (26).

Periosteal reactions occur due to tumor displacement and infiltration. Rana et al. (27) demonstrated that the pattern of periosteal reaction is highly suggestive of either an aggressive or non-aggressive process. In aggressive periosteal reactions, fast-growing masses are characterized by a Codman triangle, and spiculated (sunburst or hair-onend/perpendicular), laminated (onion skin), or disorganized features. The spiculated or Codman triangle subtypes of periosteal reaction are the most dominant types detected in the bones of the extremities (27). Compared to MRIs, CT scans have superior bony resolution and greater 

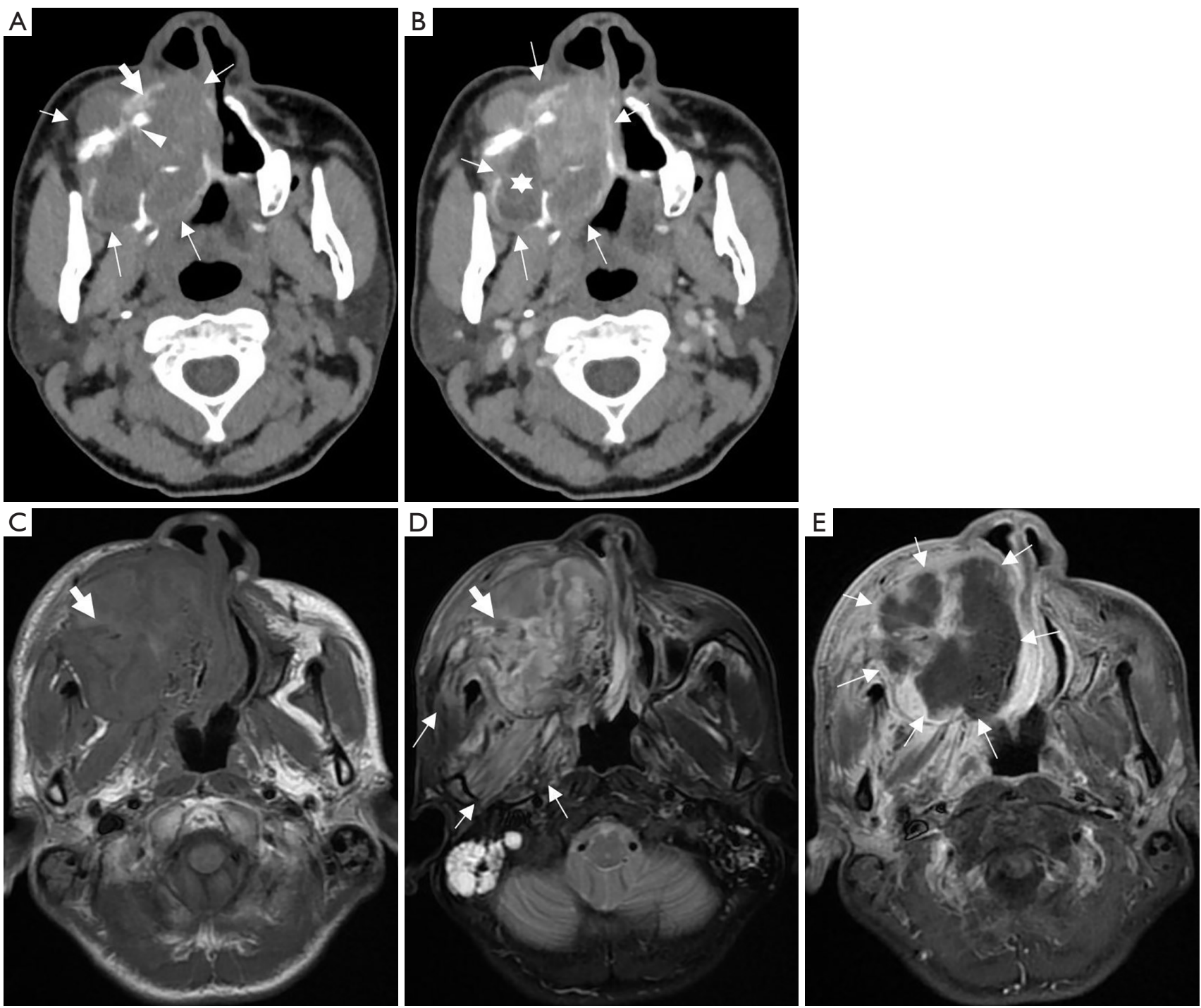

Figure 2 Medical imaging of a 39-year-old man with radiation-induced osteosarcoma of the right maxilla 3 years after nasopharyngeal carcinoma radiotherapy (histological subtype: chondroblastic). (A) An axial computed tomography (CT) image showing a mixed lytic and blastic lesion of the right maxilla with a spiculated periosteal reaction (white arrowhead), extraosseous soft-tissue extension (thin white arrows), and osteoid matrix (thick white arrow). (B) A postcontrast axial CT image showing peripheral rim enhancement of the tumor (thin white arrows). Non-enhancing areas (star) represent the chondroid matrix in the tumor. (C,D) An axial T1-weighted image (C) and a fat-suppressed axial T2-weighted image (T2WI) (D) of the soft tissue component showing heterogeneous signal intensity with patchy hypointensity (thick white arrows), suggesting osteoid matrix. T2WI showing diffuse edema in the right masseter and the internal and external pterygoids (thin white arrows). (E) A fat-suppressed axial T1-weighted contrast-enhanced image showing a peripheral rim enhancing mass. The large central areas did not enhance (thin white arrows), representing the chondroid matrix in the tumor.

performance in visualizing osseous changes, such as cortical destruction, periosteal reactions, and osteoid matrix (25). In the present study, a spiculated periosteal reaction in patients with IFBO was more common than a lamellar periosteal reaction or a Codman triangle.
While MRI is superior in depicting soft tissues and bone marrow infiltration (medulla), CT is superior in illustrating expansile lesions with regular destruction of the cortical bone. The importance of CT and MRI scans was highlighted in the current study. Moreover, T1-weighted 

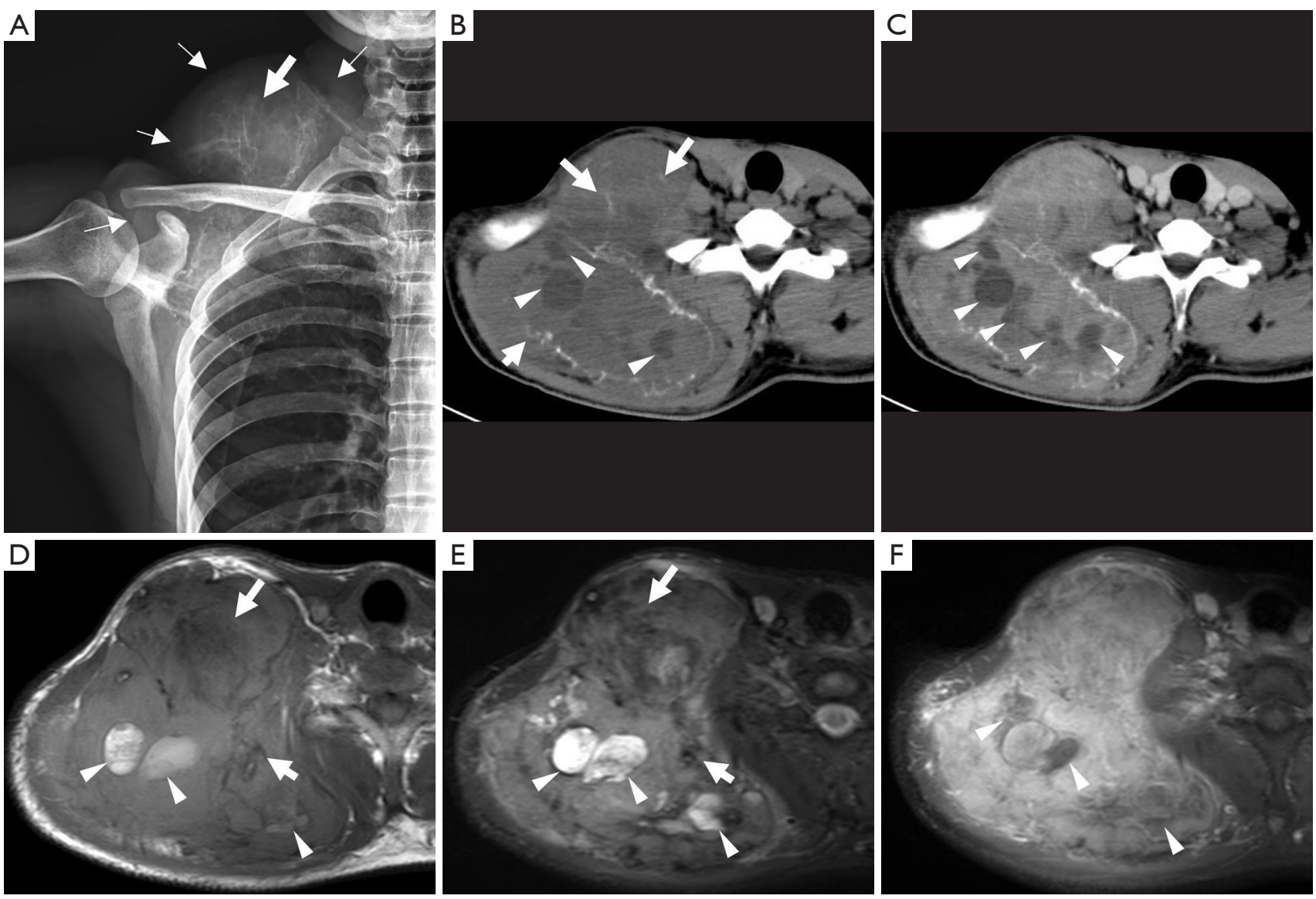

Figure 3 Medical imaging of a 32-year-old man with osteosarcoma of the right scapula (histological subtype: osteoblastic). (A) A radiograph of the right shoulder showed osteolytic bone destruction at the right scapula with osteoid matrix (thick white arrow) and a large soft tissue mass (thin white arrows). (B) An axial computed tomography (CT) image showing a large mixed density mass with cortical destruction at the right scapula. Osteoid matrix (thick white arrows) and low-density necrosis or mucous degeneration (arrowheads) are shown to occur in the mass. (C) A postcontrast axial CT scan showing an expansile osteolytic right scapula with heterogeneous enhancement of the extraosseous soft-tissue extension. Non-enhancing areas can be seen in the tumor (arrowheads). (D,E) An axial T1-weighted image (T1WI) (D) and a fat-suppressed axial T2-weighted image (T2WI) (E) of the soft tissue component showing heterogeneous signal intensity with patchy hypointensity (thick white arrows), suggestive of the osteoid matrix. Multiple patchy hyperintensities (arrowheads) in the tumor can be observed on both T1WI and T2WI. (F) A fat-suppressed contrast-enhanced axial T1-weight image showing heterogenous enhancement of the tumor. The areas of low signal intensity inside the soft-tissue portion of the tumor represents necrotic or mucoid degeneration (arrowheads).

images revealed soft tissue components of the tumor as isointense or hypointense, while on T2-weighted images, the signal intensity of soft tissue components of the tumor was classified as heterogeneous or hyperintense. The center of tumor masses was found to be accompanied by necrosis or hemorrhage, as well as an osteoid matrix $(10,28)$. In the present investigation, image enhancement patterns were correlated with tumor characteristics, such as histological characteristics, intratumoral hemorrhage, angiogenesis, and necrotic or cystic components.

In our study, the majority of patients (78.6\%) presented with high-grade tumors, and consistent with the findings of Lee et al. (6), the most common pathological type was osteoblastic. The majority of patients with IFBO exhibited an osteoid matrix on radiological examination, and in terms of diagnostic performance, CT outperformed radiography. Some of the rare pathological types, such as small-cell and giant-cell osteosarcomas, were non-specific 

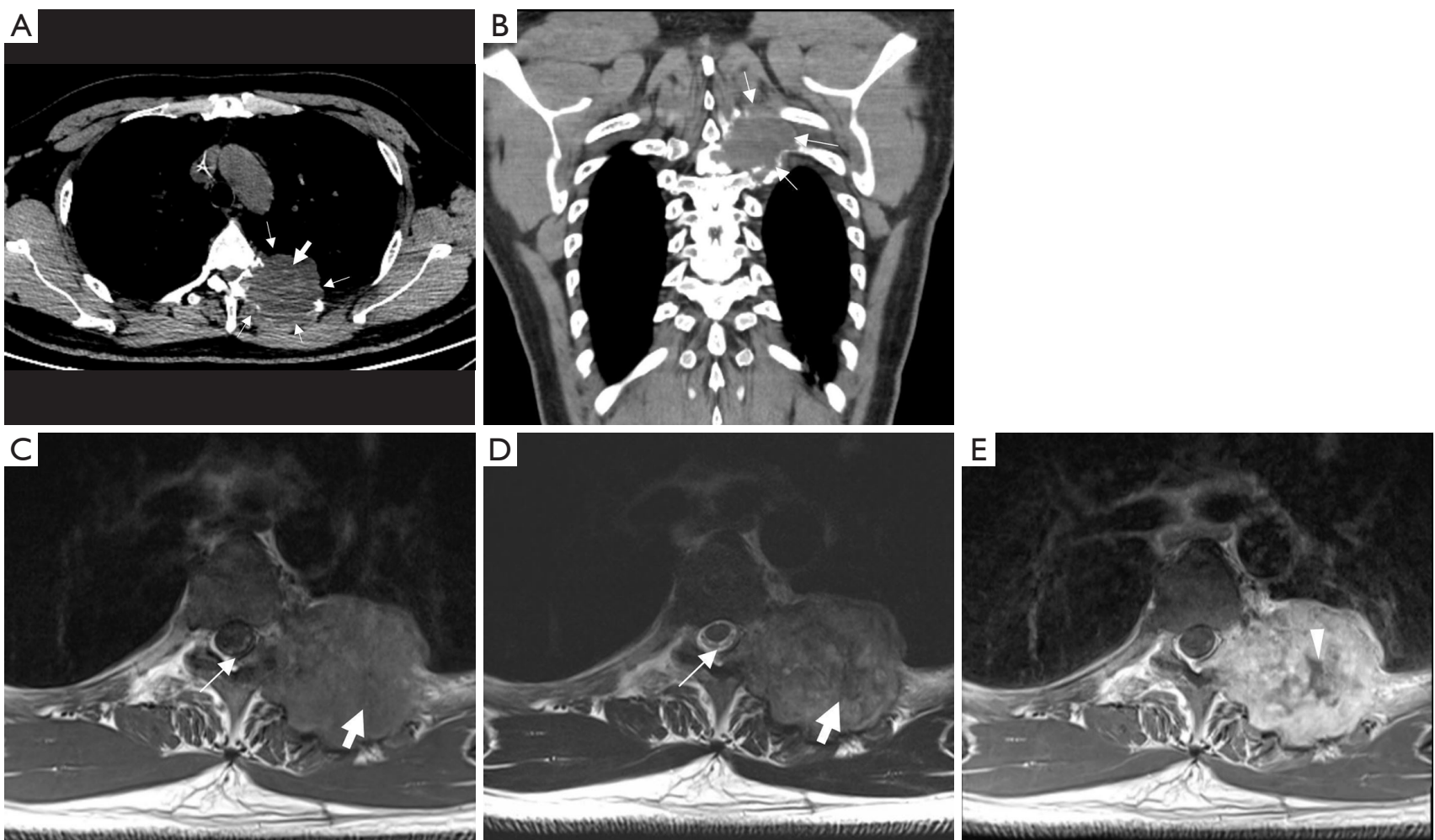

Figure 4 Medical imaging of a 37-year-old man with osteosarcoma of the left sixth rib (histological subtype: giant-cell rich). (A,B) An axial computed tomography (CT) scan (A) and a coronal 2D reconstruction (B) showed the lytic destructive mass (thin white arrows) with osteoid matrix (thick white arrow) of the left sixth rib. The mass invaded the left pedicle of the sixth thoracic vertebra and the fifth rib. (C,D) An axial T1-weighted image (C) and an axial T2-weighted image (D) showing heterogeneous signal intensity with agglomerate hypointensity (thick white arrows) of the tumor, which tends to encroach upon the left intervertebral foramen. The boundary between the tumor and the spinal cord was clear (thin white arrows). (E) An axial T1-weighted contrast-enhanced image showing a heterogeneous enhancing mass. The areas of hypointensity inside the soft-tissue portion of the tumor represent the necrotic regions (arrowhead).

on MRI scans. This lack of specificity may contribute to difficulties in differential diagnosis from other sarcomas with hyperintense and heterogeneous postcontrast enhancement. The presence of the osteoid matrix is the most important characteristic in the differential diagnosis of small-cell osteosarcoma, where small round cell tumors may histologically simulate Ewing's sarcoma. In osteosarcoma, the mineralized tumor matrix can be observed in the soft tissues or the intramedullary compartment, which contrasts with the characteristically laminated form of soft tissue mineralization in Ewing's sarcoma (29). Four cases of small-cell osteosarcomas were identified in the current study, including 2 in the ilium, 1 in the sacrum, and 1 in the lumbar spine. The osteoid matrix was observed in the soft tissues or the intramedullary compartment with the osteosarcoma, consistent with previous reports $(28,29)$.
Giant cell-rich osteosarcomas commonly involve the long bones of the extremities, and there is a paucity of literature regarding patients treated for IFBO (30-34). The diagnosis of giant cell-rich osteosarcoma is very difficult, as it mimics nonmineralized benign or malignant bone tumors. The typical radiographic pattern of giant cell-rich osteosarcoma is an ill-defined margin around a lytic lesion, with a weak periosteal reaction, and it is generally difficult to detect the soft-tissue mass (35). However, in the present research, detection of ill-defined lesions with osteolytic areas, bone expansion, destruction of the cortical bone with a soft-tissue mass, and the presence of the osteoid matrix confirmed a definitive differential diagnosis.

There were certain limitations to this retrospective study. First, although this is the largest IFBO study to date, the sample size of each type of IFBO was still small, 

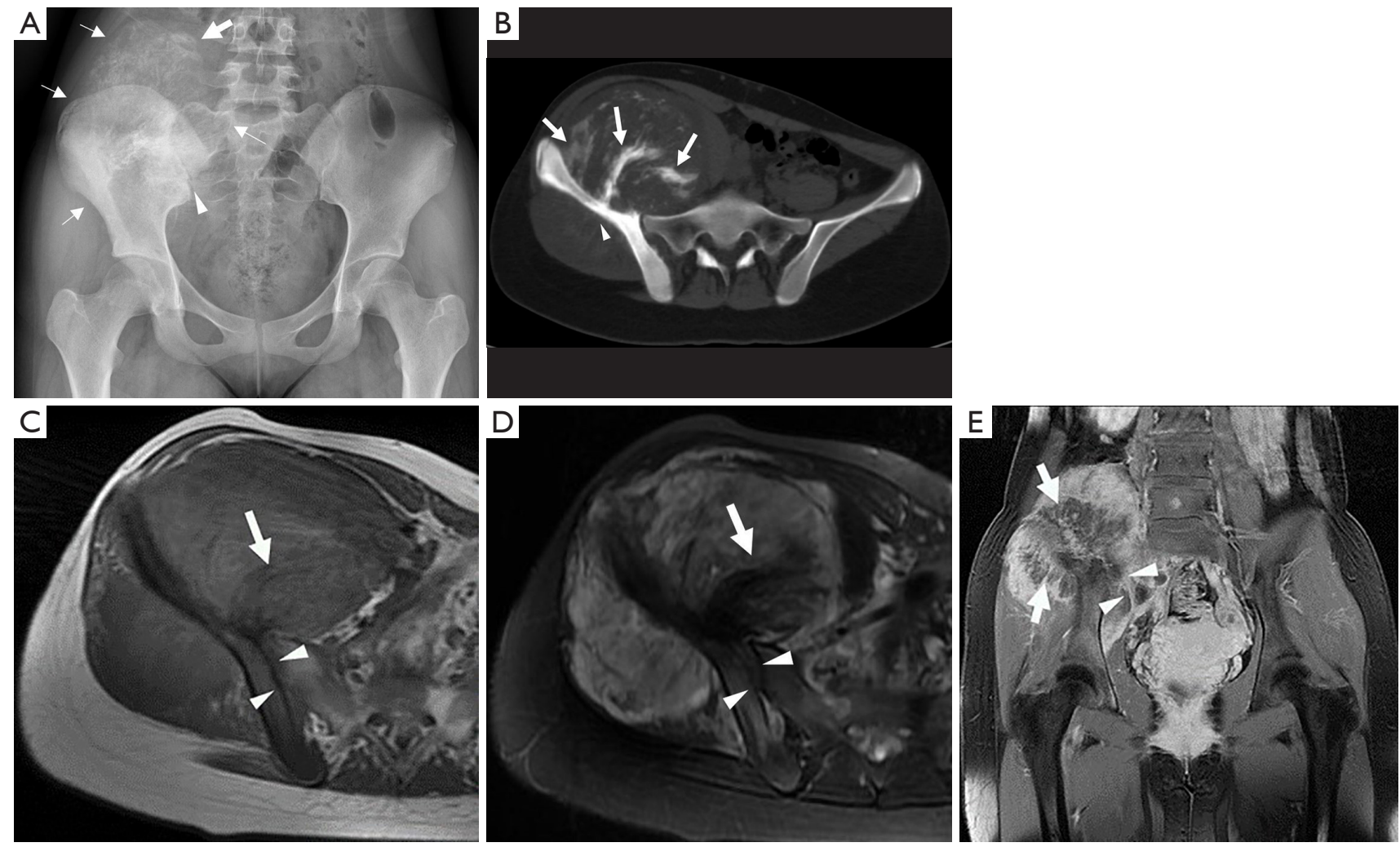

Figure 5 Medical imaging of an 18-year-old man with osteosarcoma of the right ilium (histological subtype: osteoblastic). (A) An anteriorposterior radiograph showing an osteoblastic lesion of the right ilium with a big extraosseous soft-tissue extension (thin white arrows). The osteoid matrix (thick white arrow) and right sacroiliac joint invasion (arrowhead) can be seen. (B) An axial bone algorithm computed tomography (CT) showing a mixed density mass with spotted and patchy osteoid matrix (thick white arrows) and a spiculated periosteal reaction (arrowhead). The boundary between the tumor and the adjacent muscles (right gluteus maximus and iliopsoas muscle) was unclear. (C,D) An axial T1-weighted image (C) and a fat-suppressed axial T2-weighted image (D) showing heterogeneous signal intensity with foci and patchy hypointensities (osteoid matrix, thick white arrow). The mass involved the right of the sacroiliac joint (arrowheads). (E) A fat-suppressed, contrast-enhanced coronal T1-weight image showing transarticular extension of the lesion to the right of the sacroiliac joint (arrowheads). The foci and patchy hypointensities (thick white arrows), which present the same signal intensity on $\mathrm{T} 1 \mathrm{~W}$ and $\mathrm{T} 2 \mathrm{~W}$ sequences, correspond to the osteoid matrix on the CT images.

and the images were acquired over several years. Some patients who did not receive related imaging examinations before surgery were excluded. Additionally, only patients with pathologically confirmed disease were included in this investigation, and those without pathological diagnostic confirmation were excluded, which may have led to selection bias. Second, the presence of the osteoid matrix was the most important characteristic in the diagnosis of IFBO in this study. However, this feature is not significantly different from that used in the diagnosis of typical osteosarcoma of the extremities. Furthermore, we only compared routine imaging examinations, including radiography, CT and T1weighted imaging (T1WI), T2-weighted imaging (T2WI), and contrast enhanced (CE)-T1WI sequences of MRIs. Additional quantitative imaging data with statistical analysis might provide beneficial imaging information for the diagnosis of IFBO. Third, the present series focused on the imaging features of IFBO, and no treatment strategies nor clinical outcomes were documented. Furthermore, longterm follow-up information could not be obtained for a number of patients as they had been discharged from the hospital after surgery or did not receive any treatments.

\section{Conclusions}

Although most of the IFBO images showed bone 

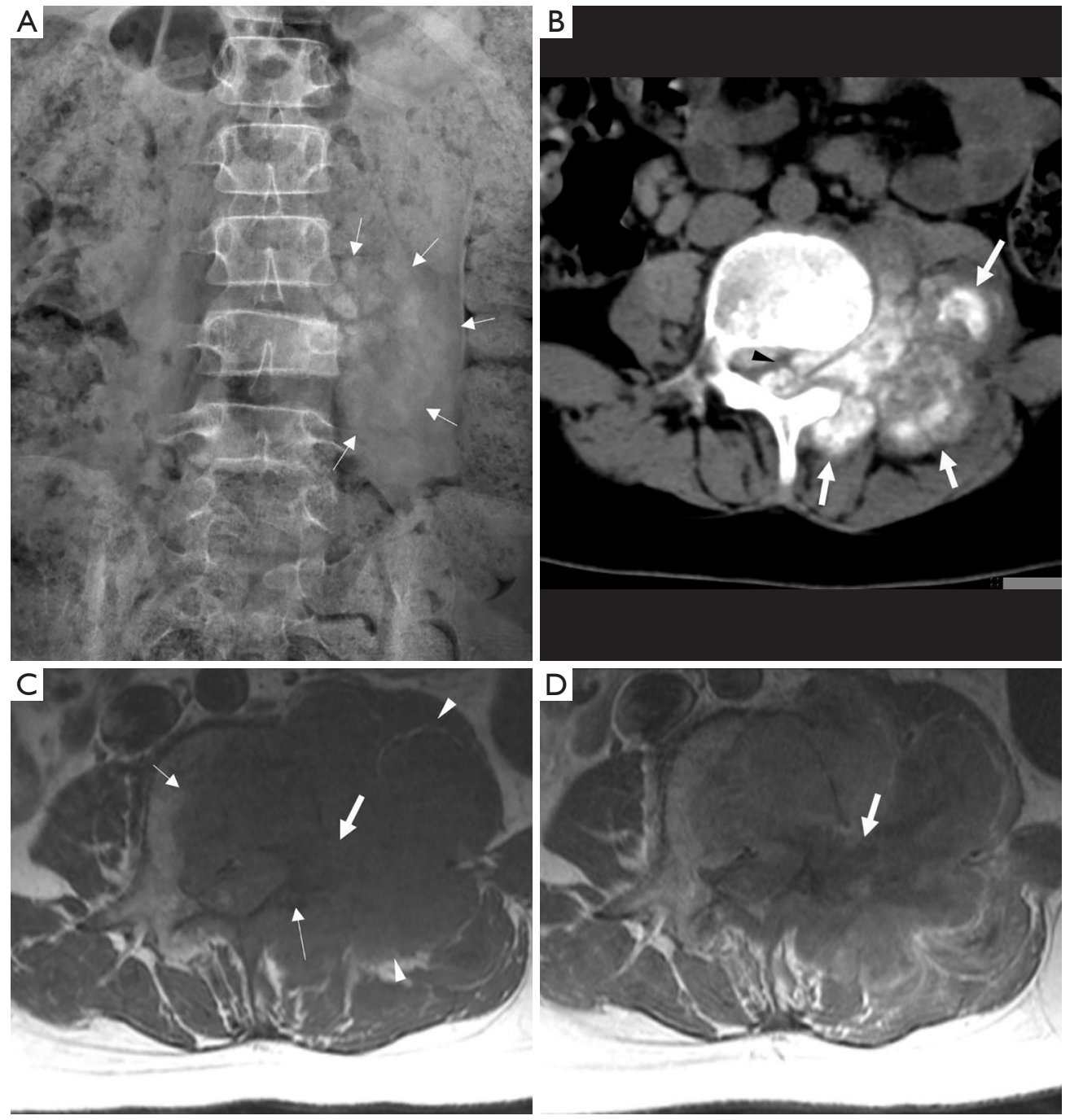

Figure 6 Medical imaging of a 12-year-old man with osteosarcoma of the third lumbar vertebra (L3) (histological subtype: osteoblastic). (A) An anterior-posterior radiograph showing a heterogeneous mass (thin white arrows) at the third lumbar vertebrae involving the vertebral body and left transverse process. (B) An axial computed tomography (CT) image showed an extension of the tumor through the left intervertebral foramen into the spinal canal. The entire lesion was hyperdense (osteoid matrix, thick white arrows), and portions of it occupied the spinal canal (black arrowhead). (C) An axial T1-weighted image showing heterogeneous signal intensity mass with a patchy hypointense (osteoid matrix, thick white arrow). The mass invaded the paravertebral muscles (the psoas muscle and the erector spinal muscle, arrowheads), the vertebral body, and the left pedicle (thin white arrows). The tumor boundary was unclear. (D) A contrast-enhanced axial T1-weight image showing a peripheral rim enhancing mass. The patchy hypointensity (thick white arrow), which presents the same signal intensity on T1W sequences, corresponds to the osteoid matrix on the CT images.

destruction with soft tissue mass and osteoid matrix, different lesion locations presented with different clinical and imaging characteristics. Maxillofacial osteosarcoma is commonly associated with a history of exposure to radiation. Pelvic osteosarcoma is more likely to invade the sacroiliac joint. Vertebral osteosarcoma frequently arises in the transverse process and the pedicle with partial vertebral body involvement. The presence of the osteoid matrix is the most important characteristic in diagnosing IFBO, even for rare subtypes of osteosarcomas, such as small-cell and giant cell-rich osteosarcomas. Nevertheless, the features of IFBO may be significantly different to those observed 
in osteosarcomas of the extremities, with the exception of a typically older age at onset for IFBO patients. Hence, recording and saving imaging and clinical characteristics will improve the accuracy and efficacy of IFBO diagnosis in these rare variants of IFBO and facilitate the differentiation of osteosarcomas from other tumors.

\section{Acknowledgments}

Funding: This study was supported by the High Level Hospital Program, Health Commission of Guangdong Province, China (HKUSZH201901026).

\section{Footnote}

Conflicts of Interest: All authors have completed the ICMJE uniform disclosure form (available at https://dx.doi. org/10.21037/qims-21-393). The authors have no conflicts of interest to declare.

Ethical Statement: The authors are accountable for all aspects of the work in ensuring that questions related to the accuracy or integrity of any part of the work are appropriately investigated and resolved. The study was conducted in accordance with the Declaration of Helsinki (as revised in 2013) and was approved by the ethics board of the University of Hong Kong-Shenzhen Hospital. Individual patient consent was waived due to the retrospective nature of this analysis.

Open Access Statement: This is an Open Access article distributed in accordance with the Creative Commons Attribution-NonCommercial-NoDerivs 4.0 International License (CC BY-NC-ND 4.0), which permits the noncommercial replication and distribution of the article with the strict proviso that no changes or edits are made and the original work is properly cited (including links to both the formal publication through the relevant DOI and the license). See: https://creativecommons.org/licenses/by-nc-nd/4.0/.

\section{References}

1. Close AG, Dreyzin A, Miller KD, Seynnaeve BKN, Rapkin LB. Adolescent and young adult oncology-past, present, and future. CA Cancer J Clin 2019;69:485-96.

2. Tabone MD, Terrier P, Pacquement H, Brunat-Mentigny M, Schmitt C, Babin-Boilletot A, Mahmoud HH, Kalifa C. Outcome of radiation-related osteosarcoma after treatment of childhood and adolescent cancer: a study of 23 cases. J Clin Oncol 1999;17:2789-95.

3. Minard-Colin V, Kalifa C, Guinebretiere JM, Brugieres L, Dubousset J, Habrand JL, Vassal G, Hartmann O. Outcome of flat bone sarcomas (other than Ewing's) in children and adolescents: a study of 25 cases. Br J Cancer 2004;90:613-9.

4. Salas S, Huynh TK, Giorgi R, Deville JL, Bollini G, Curvale G, Blesius A, Gentet JC, Bui B, Bouvier C, Duffaud F. A study of 28 flat bone osteosarcomas: prognostic factors and early and long-term outcome. Eur J Cancer Care (Engl) 2011;20:322-9.

5. Kellie SJ, Pratt CB, Parham DM, Fleming ID, Meyer WH, Rao BN. Sarcomas (other than Ewing's) of flat bones in children and adolescents. A clinicopathologic study. Cancer 1990;65:1011-6.

6. Lee JA, Kim MS, Koh JS, Kim MS, Kim DH, Lim JS, Kong CB, Song WS, Cho WH, Lee SY, Jeon DG. Osteosarcoma of the flat bone. Jpn J Clin Oncol 2010;40:47-53.

7. Pratt CB, Meyer WH, Rao BN, Pappo AS, Fleming ID, Luo X, Cain A, Kaste SC, Shearer PD, Jenkins JJ 3rd. Comparison of primary osteosarcoma of flat bones with secondary osteosarcoma of any site. Cancer 1997;80:1171-7.

8. Duffaud F, Digue L, Baciuchka-Palmaro M, Volot F, Perles-Daniel C, Garbe L, Favre R. Osteosarcomas of flat bones in adolescents and adults. Cancer 2000;88:324-32.

9. Hong JB, Cho KH, Choi JH. Periosteal osteosarcoma arising from the rib and scapula: imaging features in two cases. Korean J Radiol 2014;15:370-5.

10. Park SK, Lee IS, Cho KH, Lee YH, Yi JH, Choi KU. Osteosarcoma of pelvic bones: imaging features. Clin Imaging 2017;41:59-64.

11. Wang S, Shi H, Yu Q. Osteosarcoma of the jaws: demographic and CT imaging features. Dentomaxillofac Radiol 2012;41:37-42.

12. Cai PQ, Wu YP, Li L, Zhang R, Xie CM, Wu PH, Xu JH. CT and MRI of radiation-induced sarcomas of the head and neck following radiotherapy for nasopharyngeal carcinoma. Clin Radiol 2013;68:683-9.

13. Fletcher CD, Bridge JA, Hogendoorn PC, et al. WHO classification of tumours of soft tissue and bone. 4th edition. Lyon, France: IARC Press, 2013.

14. Mendenhall WM, Fernandes R, Werning JW, Vaysberg M, Malyapa RS, Mendenhall NP. Head and neck osteosarcoma. Am J Otolaryngol 2011;32:597-600.

15. van den Berg H, Merks JH. Incidence and grading of 
cranio-facial osteosarcomas. Int J Oral Maxillofac Surg 2014;43:7-12.

16. Argon A, Doğanavşargıl B, Ünal Yıldırım F, Sezak M, Midilli R, Öztop F. Osteosarcomas of jaw: experience of a single centre. J Plast Surg Hand Surg 2015;49:13-8.

17. Thariat J, Julieron M, Brouchet A, Italiano A, Schouman T, Marcy PY, Odin G, Lacout A, Dassonville O, PeyrottesBirstwisles I, Miller R, Thyss A, Isambert N. Osteosarcomas of the mandible: are they different from other tumor sites? Crit Rev Oncol Hematol 2012;82:280-95.

18. Pack SE, Al Share AA, Quereshy FA, Baur DA. Osteosarcoma of the Mandible Arising in Fibrous Dysplasia-A Case Report. J Oral Maxillofac Surg 2016;74:2229.e1-4.

19. Reis C, Genden EM, Bederson JB, Som PM. A rare spontaneous osteosarcoma of the calvarium in a patient with long-standing fibrous dysplasia: CT and MR findings. Br J Radiol 2008;81:e31-4.

20. Abdulrahman RE, White CS, Templeton PA, Romney B, Moore EH, Aisner SC. Primary osteosarcoma of the ribs: CT findings. Skeletal Radiol 1995;24:127-9.

21. Yalniz E, Ozcan M, Copuroglu C, Memisoglu S, Yalçin O. Osteosarcoma of the lumbar vertebra: case report and a review of the literature: rare localization with long survival. Arch Orthop Trauma Surg 2009;129:1701-5.

22. Mirabello L, Troisi RJ, Savage SA. Osteosarcoma incidence and survival rates from 1973 to 2004: data from the Surveillance, Epidemiology, and End Results Program. Cancer 2009;115:1531-43.

23. Saab R, Rao BN, Rodriguez-Galindo C, Billups CA, Fortenberry TN, Daw NC. Osteosarcoma of the pelvis in children and young adults: the St. Jude Children's Research Hospital experience. Cancer 2005;103:1468-74.

24. Rajiah P, Ilaslan H, Sundaram M. Imaging of sarcomas of pelvic bones. Semin Ultrasound CT MR 2011;32:433-41.

25. Wang CS, Yin QH, Liao JS, Lou JH, Ding XY, Zhu YB,

Cite this article as: Luo Z, Li J, Qin G, Zeng H, He Z, Pan D, Li Y, Chen W, Shen X. Clinical and imaging features of 112 patients with irregular and flat bone osteosarcoma. Quant Imaging Med Surg 2022;12(3):1988-2001. doi: 10.21037/qims-21393
Chen KM. Primary diaphyseal osteosarcoma in long bones: imaging features and tumor characteristics. Eur J Radiol 2012;81:3397-403.

26. Murphey MD, Robbin MR, McRae GA, Flemming DJ, Temple HT, Kransdorf MJ. The many faces of osteosarcoma. Radiographics 1997;17:1205-31.

27. Rana RS, Wu JS, Eisenberg RL. Periosteal reaction. AJR Am J Roentgenol 2009;193:W259-72.

28. Zhong J, Hu Y, Si L, Geng J, Xing Y, Jiao Q, Zhang $\mathrm{H}$, Yao W. Clarifying prognostic factors of small cell osteosarcoma: A pooled analysis of 20 cases and the literature. J Bone Oncol 2020;24:100305.

29. Nakajima H, Sim FH, Bond JR, Unni KK. Small cell osteosarcoma of bone. Review of 72 cases. Cancer 1997;79:2095-106.

30. Kinoshita G, Yasoshima H. Giant cell-rich tumor of the rib. J Orthop Sci 2006;11:312-7.

31. Fu HH, Zhuang QW, He J, Wang LZ, He Y. Giant cellrich osteosarcoma or giant cell reparative granuloma of the mandible? J Craniofac Surg 2011;22:1136-9.

32. Verma RK, Gupta G, Bal A, Yadav J. Primary giant cell rich osteosarcoma of maxilla: an unusual case report. J Maxillofac Oral Surg 2011;10:159-62.

33. Ha PK, Eisele DW, Frassica FJ, Zahurak ML, McCarthy EF. Osteosarcoma of the head and neck: a review of the Johns Hopkins experience. Laryngoscope 1999;109:964-9.

34. Ito Y, Sugita S, Segawa K, Sugawara T, Tsujiwaki M, Fujita H, Ono Y, Morita T, Emori M, Hasegawa T. Giant cell-rich osteosarcoma of the vertebra with murine double minute chromosome 2- and cyclin-dependent kinase 4-positive and histone H3F3A mutant p.Gly34Trpnegative immunophenotypes. Pathol Int 2018;68:324-6.

35. Wang CS, Yin QH, Liao JS, Lou JH, Ding XY, Zhu YB. Giant cell-rich osteosarcoma in long bones: clinical, radiological and pathological features. Radiol Med 2013;118:1324-34. 